\title{
BIBECHANA
}

A Multidisciplinary Journal of Science, Technology and Mathematics

ISSN 2091-0762 (online)

Journal homepage: http://nepjol.info/index.php/BIBECHANA

\section{Scientific Calculators and the Skill of Efficient Computation}

\author{
Mohd Yusuf Yasin \\ ECE Department, Integral University, Lucknow, UP, India \\ e-mail: mmyasin@rediffmail.com \\ Article history: Received 12 May 2011; Accepted 21 June 2011
}

\begin{abstract}
Calculators are part and parcel of modern education. Involvement of science and engineering in different fields of knowledge is increasing with each bit of time is passed by, and they are playing a role in description and characterization of the delicate phenomena of nature arising day by day. These fields of knowledge and mathematics in particular, are influencing even those distant branches of knowledge, which were so far imagined to be free of mathematics. Even art is not free of mathematics and there exists mathematical art. Computations are getting lengthy and complex specially in design and analysis of engineering systems. Scientific calculators are handy tools. But an efficient computation is a skill that can be developed.
\end{abstract}

Keywords: scientific calculators; calculator techniques; efficient computation; efficient use of calculators, computation skills; numerical techniques

\section{Introduction}

Today's world is of science and technology. Science has pervaded in other branches of knowledge, it has become difficult to think a subject thoroughly explained without inviting science to help. More insight into the natural phenomena are required and as a way, they demand computations. Subjects specifically related to engineering and technology, are heavily computational. Fortunately, powerful computing devices are readily available and in every body's reach. It has been observed that usually students buy scientific calculators. But it is a pity that most of these students usually imagine the operations of multiplying and dividing, and computations of functions like sine and cosine is the all a scientific calculator can do.

Today's scientific calculators are highly powerful and if their operation is properly understood, a little innovative imagination can do wonders. Long calculations are a normal part of design and analysis, where students spend time like anything, and conclude the computational procedure in distaste.

In this short paper efforts are made to point out that this attitude needs a rethinking on the process afresh to settle down the matters favorably. 


\section{Preliminaries}

A little knowledge is required before one should start working with a calculator. Every calculator is complemented with a manual, which delineates the calculator capabilities. It needs proper attention to go through the manual. Scientific calculators are highly capable and therefore are complex entities. Their functioning is grouped. Therefore a calculator needs to be prepared for a proper functioning. A very common mistake usually observed in this context is the computation performed on functions containing 'circular functions' without a care of the proper mode of their arguments or computations on functions containing a 'log' without an idea of the natural log or log on base 10. Such improper modes lead to wrong results and may cause a failure to maintain interest in the process of design and analysis both.

\section{Calculator Techniques}

Almost all scientific calculators posses a temporary register under the key Ans, which automatically modifies after each computation, and holds the current result. In design or analysis, this result is usually needed by the next step. Still there are examples where recursive computations are necessary to ascertain the final results. Normally, calculators have a few more variable like $A, B, C, \ldots$, but presently a description is restricted on some simple tricks based upon the Ans key only. In fig.1 the general scheme adopted here is presented.

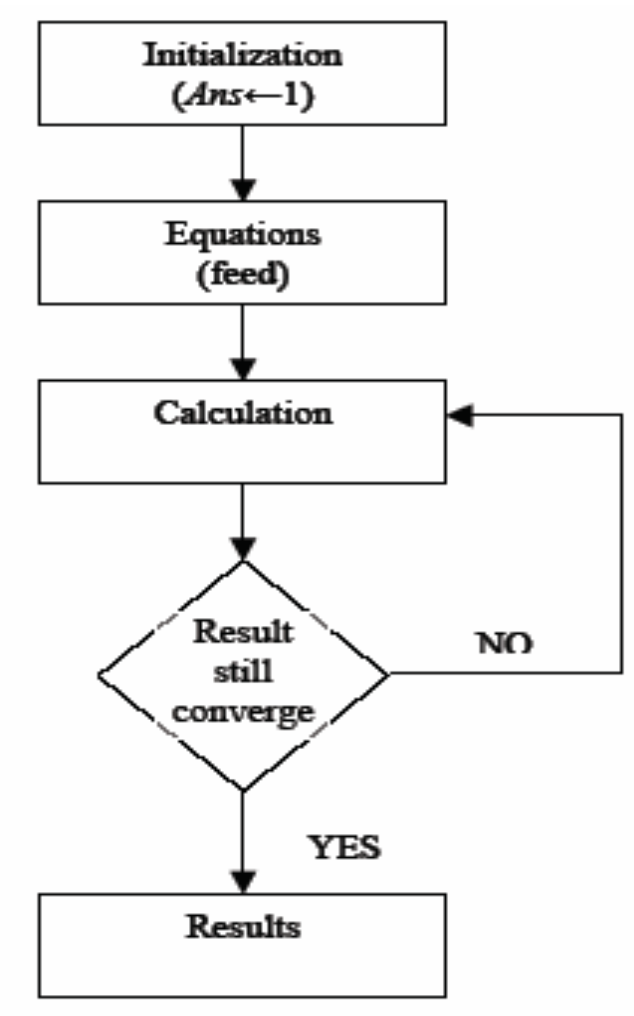

Figure 1: General flow graph of the computational procedure 
In the following sections, two examples are considered for the purpose of description on how the calculator tricks are applicable to ease the process of approaching final accurate result recursively. In both these examples, due to their simplicity and excellent bidirectional approach to the final accurate solution, the simple Newton - Raphson method is employed.

\subsection{Calculators Referred}

In this article, scientific calculators referred to as the casio make only, like fx-82MS, fx-991MS or later versions. All these calculators have a distinct feature of displaying full scientific equations before and after the calculation is done. A 'Scientific Calculator' afterwards refers to a calculator possessing these specific features. Other brands of calculators with similar features can also be used for the purpose of this article, possibly with a little or no modification in the computational scheme depending upon their operating principles [1,2].

\subsection{Example - 1: Square root of a given number}

In the first example, computation of the square root of a given number ' $a$ ' is considered. Let $x$ be the square root of the number $a$ Thus mathematically this can be represented as $x^{2}=a$, and the corresponding functional equation is $f(x)=x^{2}-a$. According to the ordinary Newton Raphson method, the square root is approached by the equation $x_{n+1}=x_{n}-\frac{f\left(x_{n}\right)}{f^{\prime}\left(x_{n}\right)}$, where $x_{n}$ is the first approximation, $x_{n+1}$ is the new improved approximation and $f^{\prime}\left(x_{n}\right)$ is the first derivative of the function $f\left(x_{n}\right)$. When applied to the required square root of the given number, this approximating equation can be expressed as [3]:

$$
x_{n+1}=1 / 2\left(x_{n}+\frac{a}{x_{n}}\right)
$$

This method is particularly important as it is quite fast in approaching the final reasonable result in just a few iterations. Further, the final result is possible even when an absurd initial approximation is adopted, of course, in such a case, total number of iterations may be increased.

For applying a scientific calculator, the above root approximating equation is considered for determining a square root of the given number 3 , with an initial approximation, say, 1 . The procedure can be as follows.

1. Type the initial approximation, (say, ' 1 ' for this example) and press ' $=$ ' (enter through the key ' $=$ ' in casio calculators, refer, fx-82MS, fx-991MS). This step stores the ' 1 ' in the register associated with the key 'Ans'. As every result is automatically stored under the key 'Ans', therefore this key can be used as a variable. Hence, the square root determining equation can be re written with 'Ans' as variable,

$$
A n s=1 / 2\left(A n s+\frac{a}{A n s}\right)
$$

Here in equation (2), the 'Ans' inside the braces refers to $x_{n}$ and contain the last result, whereas the 'Ans' outside refers to $x_{n+1}$ and automatically modifies to the latest result. As 
per the data handling scheme of the calculators, the equation (2) is read as $A n s \leftarrow 1 / 2\left(A n s+\frac{a}{A n s}\right)$, where the calculation results on the right hand side are transferred to the register attached to the 'Ans' key,

2. Second step is to type the equation in accordance with the right hand side of the above equation assuming the variable key Ans and do the required computation by just pressing the '=' key as many number of times as required. The sequence of keys pressed on a calculator may be as follows:

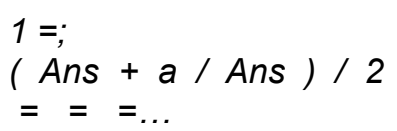

In this construct, each key is separated by multiple spaces for the sake of clarity only, the spaces are not a part of the equation. As is evident from the calculation of the examples below, for three iterations, the total number of key presses are just 14 in each case.

Convergence of $\sqrt{3}$ to it's final numerical value is as follows:

$$
\begin{aligned}
& \text { Ans } \leftarrow 1.0 \\
& \text { Ans } \leftarrow 2.0 \\
& \text { Ans } \leftarrow 1.75 \\
& \text { Ans } \leftarrow 1.732142857 \\
& \text { Ans } \leftarrow 1.73205081 \\
& \text { Ans } \leftarrow 1.732050808 .
\end{aligned}
$$

The value calculated from the calculator is $\sqrt{3}=1.732050808$, thereby showing no error in the two results. However, $\sqrt{3}=1.732$ is normally the accepted approximate value, and is available within third iteration.

\subsection{Root of an equation}

Again, consider to find the intersection of the curves [4]

$$
\begin{aligned}
& y=\cos x \text { and } \\
& y=x .
\end{aligned}
$$

It is a single root problem. A solution exists as shown in Fig. 2. Analytically, the solution is obtained as follows. Applying the ordinary Newton Raphson method, equation for solution is obtained as $f(x)=x-\cos x$ from eq. (5) above. The corresponding approximation equation to it's root is

$$
x_{n+1}=x_{n}-\frac{x_{n}-\cos x_{n}}{1+\sin x_{n}}=\frac{x_{n} \sin x_{n}+\cos x_{n}}{1+\sin x_{n}} .
$$


The computational equation for a calculator is written as given in equation (7)

$$
A n s=\frac{A n s \sin A n s+\cos A n s}{1+\sin A n s}
$$

Convergence pattern of the root of the above equation with 1.0 as initial approximation, and for 'radians' as the mode of calculation, is

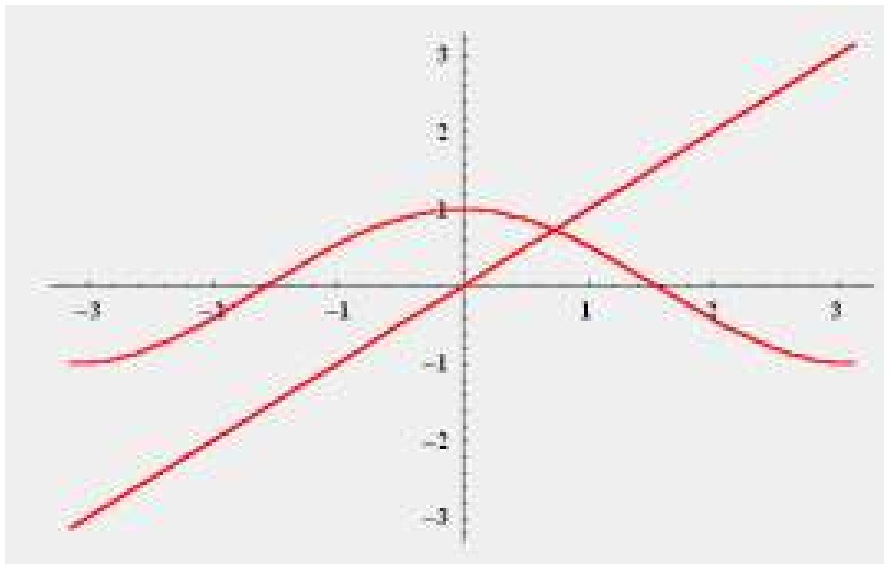

Figure 2: Graphical solution of the equations of section 3.3 [4]

$$
\begin{aligned}
& \text { Ans } \leftarrow 1 \\
& \text { Ans } \leftarrow 0.750363867 \\
& \text { Ans } \leftarrow 0.73911289 \\
& \text { Ans } \leftarrow 0.739085133 \\
& \text { Ans } \leftarrow 0.739085133
\end{aligned}
$$

A root of third decimal place accuracy is again achieved just within three iterations. It is important to consider that the concerned equations contain circular function, and therefore the selection of the mode RADIAN becomes important. The results presented above are obtained in RADIAN mode.

Purpose of the problem in equation (5) is to bring out the importance of consideration as mentioned earlier. In the solution of equation (6), 'Deg' mode selection will produce a look alike root, 0.999847784 , which unfortunately is not a root but merely an approximation of the equation (5) for the argument of the circular function tending $0(\cos x ; x \rightarrow 0)$. This situation arises due to an inappropriate mode selection.

\section{Discussions}

The above examples are common yet simple and are readily available in text books on Advanced Mathematics for Engineers and Scientists, Numerical Techniques etc. here the point of emphasis is how to make the computation effectively. The effective computational process for example in section 3.2 for a single digit number as per the flow graph, needs, all in all, 14 judicial key presses 
on an already ON calculator. However, the engineering students have often been observed haggling for hours over solving such problems and with absurd or at least, inaccurate come outs.

\section{Conclusion}

For fields like numerical methods, where usually computations are huge but are iterative in nature, it is important to optimize computational efforts. Such efforts are based on computational skills. These are some of the calculator tricks presented here which can be found quite useful. Scientific calculators are quite powerful, but their real power remains untapped. A little practice can help develop the capability to perform computations in technical manner.

\section{References}

[1] Users' Guide, fx-82MS/83MS/85MS/270MS/300MS/350MS, http://support.casio.com

[2] Users' Guide, fx-95MS/100MS/115MS/(912MS)/570MS/991MS, http://support.casio.com

[3] Erwin Kreyszig, Advanced Engineering Mathematics, $5^{\text {th }}$ edition, Wiley Eastern Limited, 1989, pp 764

[4] http://www.sosmath.com/calculus/diff/der07/der07.html; Graphical solution for the equations given in eq. (5) 